\title{
Deep cerebral vein thrombosis due to iron deficiency anemia: A case report
}

\author{
Nao Nishida, Yoshimasa Sueda, Tomomi Kanbara, Takashi Kurashige, Tsuyoshi Torii* \\ Department of Neurology, Natonal Hospital Organization Kure Medical Center, Japan
}

Received: April 20, 2016

DOI: $10.5430 /$ crim.v3n3p4
Accepted: May 30, $2016 \quad$ Online Published: June 1, 2016

URL: http://dx.doi.org/10.5430/crim.v3n3p4

\begin{abstract}
We report on a 28-year-old woman who developed deep cerebral vein thrombosis (CVT) associated with iron deficiency anemia (IDA). Activation of platelet function by IDA may lead to CVT and sometimes results in death when a deep central vein is affected. While IDA is a common condition, especially among young women, proper management of IDA is needed to prevent severe venous thrombosis.
\end{abstract}

Key Words: Cerebral vein thrombosis, Iron deficiency anemia, Platelet function, Magnetic resonance venography, Susceptibilityweighted imaging

\section{INTRODUCTION}

A 28-year-old woman developed deep cerebral vein thrombosis (CVT) associated with iron deficiency anemia (IDA). Activation of platelet function by IDA may lead to CVT and sometimes results in death when a deep central vein is affected. IDA is a common condition, particularly among young women, and should be managed properly.

\section{Case presentation}

A 28-year-old woman was admitted to our hospital with a 2-day-history of progressive vomiting, disturbance of consciousness, and abnormal behavior such as wearing her children's clothes. She had taken oral contraceptives (OCPs) from age 16 to 19 years old because of IDA and dysmenorrhea. She had two children, and no history of miscarriages. Her menstrual cycle was irregular, with heavy blood loss. She had no family history of coagulation abnormalities or allergies. On admission, she was unable to follow verbal instructions. She had a Glasgow coma scale score of $7(\mathrm{E} 4 \mathrm{~V} 1 \mathrm{M} 2)$ and body temperature was $37.9^{\circ} \mathrm{C}$. Invol- untary, bilateral, medial, and inferior deviation of the eyes was observed. Involuntary movements were seen, along with occasional decerebrate posture. Head computed tomography (CT) initially showed bleeding in the third cerebral ventricle, enlargement of the lower angle of the lateral cerebral ventricles bilaterally, and bilateral low-density lesions at the head of the caudate nucleus, lenticular nucleus, and thalamus. These same regions appeared hyperintense on T2-weighted imaging, and isointense on diffusion-weighted imaging. Susceptibility-weighted imaging and magnetic resonance (MR) venography revealed deep CVT of the straight sinus, great cerebral vein, and deep middle cerebral vein (see Figure 1). Lumbar puncture was performed immediately after brain $\mathrm{CT}$; there was no elevation of protein or pleocytosis.

Laboratory tests revealed hypochromic microcytic anemia (hemoglobin $7.5 \mathrm{~g} / \mathrm{dl}$, mean corpuscular volume $59.5 \mathrm{fl}$, and a normal platelet count of $204,000 / \mu 1$ ). Serum iron and ferritin concentrations were $10 \mu \mathrm{g} / \mathrm{dl}$ and $2.4 \mathrm{ng} / \mathrm{dl}$, respectively. Prothrombin time was $12.4 \mathrm{sec}$ (INR 1.07), thrombin time was

*Correspondence: Tsuyoshi Torii, MD; Email: toriit@kure-nh.go.jp; Address: 3-1 Aoyama, Kure, Hiroshima 737-0023, Japan. 
$48 \%$, partial thromboplastin time (PTT) was $28.1 \mathrm{sec}$ (normal range is 26.9-38.1 sec), and fibrinogen was $186 \mathrm{mg} / \mathrm{dl}$. Activated protein $\mathrm{C}$ resistance, protein $\mathrm{C}$, and protein $\mathrm{S}$ were within the normal range. Lupus anticoagulant (dilute Russell viper venom test), anticardiolipin antibody $\mathrm{IgG}$, and beta-2 glycoprotein antibodies were all negative. Tests for other serum antibodies, including antinuclear and anti-DNA antibodies, showed no abnormalities. Thoracic and abdominal CT was performed to investigate the source of anemia, but no abnormalities were evident.

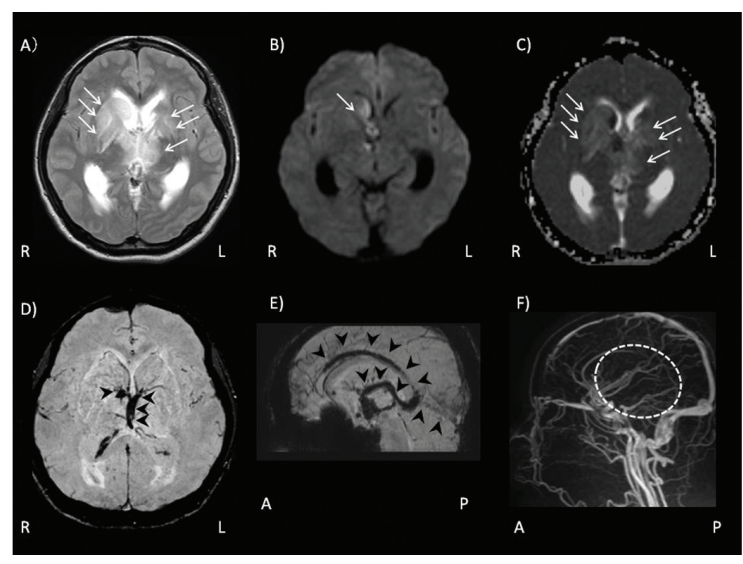

Figure 1. Brain MRI and MR venography

The patient was administered heparin for anticoagulation and acyclovir was administrated as empiric therapy until the reverse transcription PCR assay for herpes simplex virus (HSV) proved negative, because initial differential diagnosis of the patient included HSV encephalitis. Assay results for HSV antibodies were negative in both serum and cerebrospinal fluid. Brain edema exacerbated without hematoma formation in the brain parencyma and apnea developed 6 hours after arriving at our hospital, requiring mechanical ventilation and gricerol was administrated intravenously for prevention of brain edema. The patient died 20 days after admission to our hospital. Autopsy did not performed.

Figure 1 depicts the high signal intensity area (arrow) of the caudate nucleus and thalamus on the T2 weighted image (A). The apparent diffusion coefficient map (B) shows a high signal whereas an iso-signal intensity area is seen in diffusion weighted imaging (C). Susceptibility-weighted imaging (SWI) revealed stasis of the inferior sagittal sinus, internal cerebral vein of Galen, and straight sinus (arrow head) (D, E), which are not visible (dotted circle) on MR venography (F).

\section{Discussion}

CVT is a rare condition compared with arterial stroke, with an estimated prevalence of about $0.5 \%$ among patients with

Published by Sciedu Press cerebrovascular disease. Risk factors for CVT are age $<50$ years, female, prothrombotic conditions, infectious diseases, inflammatory diseases, malignancy, hematologic disorders, pregnancy and puerperium, and other disorders such as dehydration and nephrotic syndrome. ${ }^{[1,2]}$ Although the current patient had taken OCPs, they were discontinued 9 years before CVT occurred; therefore, OCPs did not affect her hypercoagulable state. Among CVT patients, $10 \%$ develop obstruction of the deep cerebral vein. The mortality rate of CVT is reportedly $3 \%-6 \%$ within 30 days after onset. Age > 37 years, male sex, disturbance of consciousness, GCS score $<9$, deep venous system thrombosis, intracranial hemorrhage, any malignancy, and infection of the central nervous system are risk factors for worsened outcome. ${ }^{[1]}$

Although the precise mechanisms involved remain unclear, poor prognosis for the present case was predictable based on the low GCS score, deep venous system thrombosis, and intracranial hemorrhage. Several etiological hypotheses have been proposed. First, deficiency of iron, which regulates thrombopoiesis, leads to decreased inhibition of thrombocytosis. Second, IDA may contribute to altered blood flow patterns because of reduced deformability of red cells and hyperviscosity. Furthermore, hypoxia caused by iron deficiency anemia causes a mismatch between increased metabolic demand in brain tissue and low hemoglobin during situations of metabolic stress, such as infection. ${ }^{[3]}$ Finally, iron deficiency induces secondary thrombocytosis and platelet activation. ${ }^{[4]}$ Akins et al. described abnormal platelet activation and function as more important than absolute platelet count in thrombus formation. ${ }^{[5]}$ In the current case, there was no observed abnormality in coagulation tests, including PTT and platelet count; therefore, abnormal platelet activation or function might have contributed to CVT.

Diagnosis of CVT is difficult if physicians fail to suspect it from a patient's history and general or neurological findings. MRI and MR venography are powerful tools for screening and diagnosis of CVT. Bilateral basal ganglia and thalamus are often affected. A hypointensity area of SWI suggests thrombus in dural sinus and cortical venous thrombosis in the acute stage. ${ }^{[1,2]}$

Treatment of CVT in the acute phase includes anticoagulation therapy, prevention of intracranial hypertension (seizure, cerebral herniation), and management of the cause of CVT. Direct endovascular thrombolysis can remove thrombus, but is complicated and indications currently have not been established. ${ }^{[2]}$ In our case, the neurological symptoms progressed too rapidly to perform intervention therapy. Despite bleeding in the third ventricle, heparin was selected for initial therapy because the bleeding was of a small amount and not seen 
in the cerebral parencyma in MRI and CT revealed brain edema without hematoma in follow-up CT. Guidelines of the American Heart Association (AHA) shows that initial anticoagulation therapy is commonly used despite the presence of hemorrhagic infarction at the time of diagnosis of CVT. ${ }^{[1]}$ Meta analysis of two randomized control studies revealed that anticoagulation therapy in the acute phase of CVT is not related to an increased risk of death regardless of the presence of intracranial hemorrhage. ${ }^{[1]}$ Our case showed bleeding only in the third cerebral ventricle, thus it was appropriate to administrate heparin intravenously.

CVT associated with IDA is a very rare condition. We reviewed 12 previous cases of CVT due to IDA; 6 of the 10 patients had obstruction of the great vein of Galen, and death occurred in 1 of the 6 cases. $^{[3,6]}$ A comparison of whole blood cell counts between patients with CVT and healthy controls revealed that severe anemia $(\mathrm{Hb}<9.0 \mathrm{~g} / \mathrm{dl})$ was significantly more frequent in the CVT patients. ${ }^{[6]}$ To our knowledge, this is the second report of death associated with obstruction of a deep cerebral vein in an IDA patient. Progressive brain edema and herniation made it difficult to treat this condition in a timely fashion.

In conclusion, whereas IDA is a common condition particularly among young women, when managed improperly, CVT, a life threatening disease, can occur. In patients with anemia and deteriorating consciousness, CVT should be considered.

\section{REFERENCES}

[1] Saposnik G, Barinagarrementeria F, Brown RD Jr, et al. American Heart Association Stroke Council and the Council on Epidemiology and Prevention. Diagnosis and management of cerebral venous thrombosis: a statement for healthcare professionals from the American Heart Association/American Stroke Association. Stroke. 2011; 42(4): 1158-1192. http://dx.doi.org/10.1161/STR.0b013e3 1820 a8364

[2] Ferro JM, Canhao P. Stroke: Pathophysiology, Diagnosis, and Management, sixth edition. Elsevier. 2016; 45: 716-729.

[3] Balci K, Utku U, Asil T, et al. Deep cerebral vein thrombosis associated with iron deficiency anaemia in adults. J Clin Neurosci. 2007; 14(2): 181-184. http://dx.doi.org/10.1016/j.jocn.2005.0 9.020
[4] Voudoukis E, Karmiris K, Oustamanolakis P, et al. Association between thrombocytosis and iron deficiency anemia in inflammatory bowel disease. Eur J Gastroenterol Hepatol. 2013; 25(10): 1212-6. http://dx.doi.org/10.1097/MEG.0b013e328363e354

[5] Akins P, Gleen S, Nemeth P, et al. Carotid Artery thrombus associated with severe iron-deficiency anemia and thrombocytosis. Stroke. 1996; 27(5): 1002-1005. http://dx.doi.org/10.1161/01. STR . 27.5 .1002

[6] Stolz E, Valdueza JM, Grebe M, et al. Anemia as a risk factor for cerebral venous thrombosis? An old hypothesis revisited. J Neurol. 2007; 254(6): 729-734. http://dx.doi.org/10.1007/s00 415-006-0411-9 